DOI: $10.3901 / J M E .2019 .09 .071$

\title{
粗糙表面湍流润滑的随机模型*
}

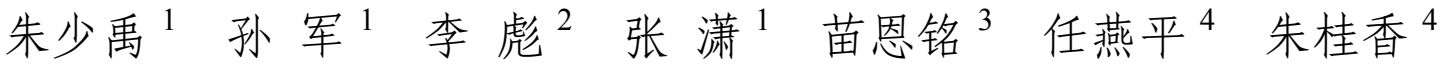 \\ (1. 合肥工业大学汽车与交通工程学院 合肥 230009; \\ 2. 合肥工业大学机械工程学院 合肥 230009; \\ 3. 合肥工业大学仪器科学与光电工程学院 合肥 230009 ; \\ 4. 潍柴动力股份有限公司 潍坊 261001)
}

\begin{abstract}
摘要: 目前粗粘表面的湍流润滑分析一般都采用各向同性粗粘表面的湍流润滑模型, 但是该模型的适用范围过于狭窄, 不适 合用于处理具有方向性特征的粗糙表面湍流润滑问题。基于 Christensen 的随机层流润滑理论和零方程形式的滑动轴承湍流润 滑理论, 推导提出了适用于粗粘表面径向滑动轴承湍流润滑分析的随机湍流润滑理论模型。该理论模型包含三部分: 一维纵 向粗糙表面径向滑动轴承的随机湍流润滑理论模型、一维横向粗糙表面径向滑动轴承的随机湍流润滑理论模型和各向同性粗 粘表面径向滑动轴承的随机湍流润滑理论模型。应用提出的随机湍流润滑模型对均匀各向同性粗粘表面径向滑动轴承湍流润 滑进行了分析, 结果与相应的试验数据一致性良好。提出的随机湍流润滑理论模型可以方便地用于具有方向性特征的粗粘表 面湍流润滑分析。此外, 提出的随机湍流润滑理论模型不仅适用于径向滑动轴承, 而且也适用于滑块和推力轴承等摩擦副机 械零件的湍流润滑研究。
\end{abstract}

关键词: 流体动力润滑; 湍流流动; 粗䄳表面; 随机理论

中图分类号: TH117

\section{Stochastic Models for Turbulent Lubrication of Rough Surfaces}

\section{ZHU Shaoyu $^{1}$ SUN Jun $^{1} \quad$ LI Biao $^{2}$ ZHANG Xiao ${ }^{1}$ MIAO Enming ${ }^{3}$ REN Yanping ${ }^{4}$ ZHU Guixiang ${ }^{4}$}

(1. School of Automotive and Transportation Engineering, Hefei University of Technology, Hefei 230009;

2. School of Mechanical Engineering, Hefei University of Technology, Hefei 230009;

3. School of Instrument Science and Optoelectronic Engineering, Hefei University of Technology, Hefei 230009;

4. Weichai Power Company Limited, Weifang 261001)

\begin{abstract}
Current turbulent lubrication analysis of rough bearing surfaces generally uses a turbulent lubrication model of an isotropic rough bearing surface. However, the scope of the model is too narrow and is not suitable for solving turbulent lubrication problems on rough bearing surfaces with directional characteristics. Based on the stochastic laminar lubrication theory of Christensen and the turbulent lubrication theory in the form of zero equation for journal bearings, stochastic turbulent lubrication theoretical models suitable for turbulent lubrication analysis of rough surface journal bearings are derived. The theoretical models consist of three parts: Stochastic turbulent lubrication theory model for one-dimensional longitudinal rough surface journal bearing, stochastic turbulent lubrication theory model for one-dimensional transverse rough surface journal bearing and stochastic turbulent lubrication model for isotropic rough surface journal bearing. The turbulent lubrication of the isotropic rough surface journal bearing is analyzed by the proposed stochastic turbulent lubrication model. And good agreement is obtained between the theoretical results and the corresponding experimental data. The proposed stochastic turbulent lubrication theoretical model can be conveniently applied to turbulent lubrication analysis of rough bearing surfaces with directional characteristics. Moreover, the theoretical models of stochastic turbulent lubrication are not only suitable for journal bearings, but also for turbulent lubrication research of friction pairs, such as sliders and thrust bearings.
\end{abstract}

Key words: hydrodynamic lubrication; turbulent flow; rough surface; stochastic theory

* 内燃机可靠性国家重点实验室开放基金资助项目(skler-201708)和国家 自然科学基金资助项目(51490660/51490661)。20180411 收到初稿, 20190203 收到修改稿 


\section{0 前言}

随着现代机械设备往大型化(如汽轮发电机 组)、高速化(如透平机)发展, 其内部的重要支撑零 部件滑动轴承工作常处于湍流润滑状态 ${ }^{[1-2]}$ 。另外, 一些特殊用途装备(如航空发动机燃油原、核主㬌等) 中的滑动轴承由于使用航空煤油和水等低黏度流体 作为润滑剂, 工作也常处于湍流润滑状态 ${ }^{[3-4]}$ 。目前, 在滑动轴承湍流润滑的理论研究中, 被普遍采用的 零方程模型 (如基于 Prandtl 混合长度理论的 Constantinescu 零方程模型 ${ }^{[5]}$ 、基于壁面定律及 Boussinesq 浴黏假设的 Ng-Pan-Elrod 零方程模型 ${ }^{[6-7]}$ 和基于壁面剪应力与相对壁面平均流速之间经验关 系的 Hirs 零方程模型 ${ }^{[8]}$ 等) 一般都认为滑动轴承表 面是光滑的。然而, 加工形成的实际滑动轴承表面 为粗粘表面, 虽然表面粗粘度相比轴承间隙较小, 但是轴承一般都工作在较大偏心率下, 此时表面粗 楉度与轴承最小油膜厚度基本处于同一数量级, 表 面粗糙度将对轴承的湍流润滑状态产生明显影响, 因此轴承表面是光滑的假定显然不符合实际情况。 而且, 在轴承工作较长时间后, 磨损、冲击损伤、 外来颗粒、空穴腐蚀及生锈等也会造成明显的轴承 表面粗糙效应。考虑到轴承实际表面粗粘因素的影 响, HASHIMOTO 等 ${ }^{[9]}$ 基于湍流边界层内的对数速 度分布律和 Hirs 的整体流动理论导出了计入均匀各 向同性粗糙表面影响的湍流润滑理论, 并以此为基 础展开了一系列研究 ${ }^{[10-12]}$ 。随着随机方法、直接法 及均一法等分析表面粗粘方法的不断涌现发展 ${ }^{[13-16]}$, 相应的分析表面粗䊁下的湍流润滑方法也在发展,

LAHMAR 等 ${ }^{[17]}$ 基于均一法分析了周期性各向同性 粗䊁表面的湍流润滑。

但是, 目前考虑表面粗粘影响的湍流润滑模型 都只能适用于分析各向同性粗粮表面的湍流润滑, 不能处理实际应用中存在的其他类型粗䊁表面(如 实际中较多采用的由磨削加工轴颈外表面和镗削加 工轴瓦内表面构成的纵向粗䊁表面滑动轴承)的湍 流润滑问题。本文针对目前粗糙表面湍流润滑模型 存在的不足, 从 Christensen 的粗糙表面随机层流润 滑理论和经典的光滑表面零方程湍流润滑模型出 发, 将 Christensen 的随机思想应用到粗糙表面的湍 流润滑中, 推导一维纵向粗粘表面的湍流润滑模型、 一维横向粗粘表面的湍流润滑模型和各向同性粗粘 表面的湍流润滑模型, 使粗糙表面滑动轴承的湍流 润滑分析更加接近实际。

\section{1 数学模型}

\section{1 粗粮表面的平均 Reynolds 型湍流润滑方程}

\subsection{1 粗䊁表面任一局部位置的湍流润滑描述}

文献[18]基于现有的零方程湍流润滑模型(不包 含 Hirs 零方程湍流润滑模型)、两方程湍流润滑模 型、代数雷诺应力湍流润滑模型和复合型湍流润滑 模型的理论计算结果与相应试验结果的对比研究, 发现零方程模型中 Ng-Pan 湍流润滑模型和复合型 湍流润滑模型的计算结果与试验结果的一致性最 好, 其余湍流润滑模型的计算结果与试验结果都有 一定的偏差。考虑到实际使用的方便性, 分析中光 滑表面滑动轴承的湍流润滑模型应用零方程湍流润 滑模型。考虑到 Hirs 零方程模型存在一定的理论缺 陷 ${ }^{[19]}$, 应用的零方程湍流润滑模型不包含 Hirs 零方 程模型。

光滑表面滑动轴承的零方程湍流润滑模型 ${ }^{[4]}$ 为

$$
\frac{\partial}{\partial x}\left[\frac{h^{3}}{\mu k_{x}} \frac{\partial p}{\partial x}\right]+\frac{\partial}{\partial z}\left[\frac{h^{3}}{\mu k_{z}} \frac{\partial p}{\partial z}\right]=\frac{V}{2} \frac{\partial h}{\partial x}+\frac{\partial h}{\partial t}
$$

式中, $p$ 为油膜压力; $h$ 为光滑表面间的油膜厚度; $V$ 为相对运动表面的切向相对速度; $\mu$ 为润滑剂动力 黏度; $x$ 坐标方向为运动表面的相对运动方向; $z$ 坐标方向既垂直于油膜厚度方向也垂直于运动表 面的相对运动方向; $t$ 为时间; $k_{x}$ 和 $k_{z}$ 为湍流润滑 方程系数。

$$
\begin{aligned}
& k_{x}=12+a_{1} R e^{b_{1}} \\
& k_{z}=12+a_{2} R e^{b_{2}}
\end{aligned}
$$

式中, $a_{1} 、 a_{2} 、 b_{1}$ 和 $b_{2}$ 为湍流润滑模型决定的参数, 如表 1 所示; $R e=\rho V h / \mu$ 为当地雷诺数, 其中 $\rho$ 为 润滑剂密度。

表 1 湍流润滑模型参数值

\begin{tabular}{ccccc}
\hline 湍流润滑模型 & $a_{1}$ & $b_{1}$ & $a_{2}$ & $b_{2}$ \\
\hline Constantinescu & 0.0260 & 0.8265 & 0.0198 & 0.7410 \\
Ng, Pan, and Elrod & 0.0136 & 0.9 & 0.0043 & 0.96 \\
\hline
\end{tabular}

湍流润滑方程式(1)的应用范围为 $R e \geqslant 1000$ 。当 $R e<1000$ 时, $k_{x}=12, k_{z}=12$, 方程式(1)即转变为 层流润滑方程 ${ }^{[4]}$ 。

对于粗粘表面滑动轴承的湍流润滑, 在轴承粗 䊁表面的任一局部位置都应满足湍流润滑方程式 (1)。这样, 结合粗䊁表面滑动轴承油膜厚度的具体 表示, 可以得到粗糙表面滑动轴承任一局部位置的 湍流润滑方程为 


$$
\frac{\partial}{\partial x}\left[\frac{H^{3}}{\mu K_{x}} \frac{\partial p}{\partial x}\right]+\frac{\partial}{\partial z}\left[\frac{H^{3}}{\mu K_{z}} \frac{\partial p}{\partial z}\right]=\frac{V}{2} \frac{\partial H}{\partial x}+\frac{\partial H}{\partial t}
$$

式中, $K_{x} 、 K_{z}$ 为新湍流润滑方程系数; $H$ 为真实油 膜厚度(图 1)。

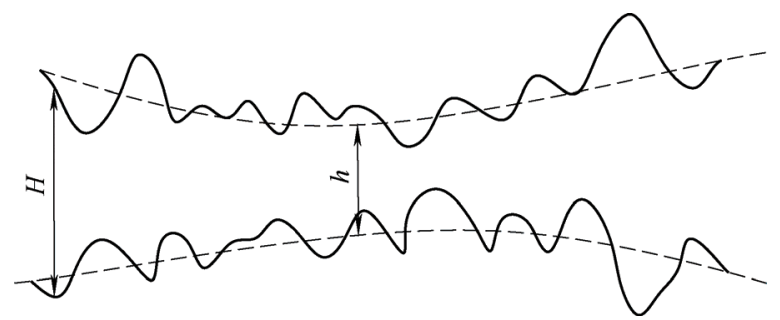

图 1 粗糙表面油膜厚度示意图

$K_{x} 、 K_{z}$ 通过将 $k_{x} 、 k_{z}$ 表达式中的 $h$ 用 $H$ 替代得 到, 为

$$
\begin{gathered}
K_{x}=12+a_{1} \operatorname{Re}^{b_{1}}\left(\frac{H}{h}\right)^{b_{1}} \\
K_{z}=12+a_{2} \operatorname{Re}^{b_{2}}\left(\frac{H}{h}\right)^{b_{2}}
\end{gathered}
$$

真实油膜厚度 $H$ 可以表示为

$$
H=h(x, z, t)+h_{s}(x, z, \xi)
$$

式中, $h$ 为名义光滑表面间的油膜厚度; $h_{s}$ 为由于 表面粗䊁的影响使真实油膜厚度 $H$ 偏离名义油膜厚 度 $h$ 的值(称为油膜厚度分量), 一般情况下假定 $h_{s}$ 沿整个轴承润滑表面的期望值为 0 。

对于任一粗粘表面, $h_{s}$ 是坐标 $x 、 z$ 及随机变 量 $\xi$ 的函数。假定随机变量 $\xi$ 的一个具体取值是从 大量有相同统计特性的相似但不完全一样的表面 粗糙高度分布情况中选择一个具体的表面粗糙高 度分布情况。这样, 在给定的 $\xi$ 值(即一个给定的 粗䊁高度分布)下, $h_{s}$ 是一个关于坐标量 $x$ 和 $z$ 的 确定性函数 ${ }^{[13]}$ 。故而, $H$ 可以被当成各态历经性 的平稳随机过程。

\subsection{2 粗䊁表面整体的湍流润滑描述}

以上基于光滑表面滑动轴承的湍流润滑模型, 结合粗䊁表面的具体表示, 得到了粗楉表面滑动轴 承的湍流润滑方程式(4)。但是, 该方程只是针对粗 糙表面滑动轴承任一局部位置湍流润滑情况的描 述, 不能直接描述粗䊁表面滑动轴承整体的湍流润 滑情况, 因此一般无法在粗粘表面滑动轴承的湍流 润滑分析中直接应用。

下面通过任取的微元控制体, 建立描述粗䊁表 面滑动轴承整体湍流润滑的平均 Reynolds 型湍流润 滑方程。

图 2 所示为一个由底面积 $\Delta x \Delta z$ 和高 $H$ 构成的控 制体, 其尺寸远小于润滑油膜总体尺寸, 但是包含
足够多的微凸峰和凹谷。

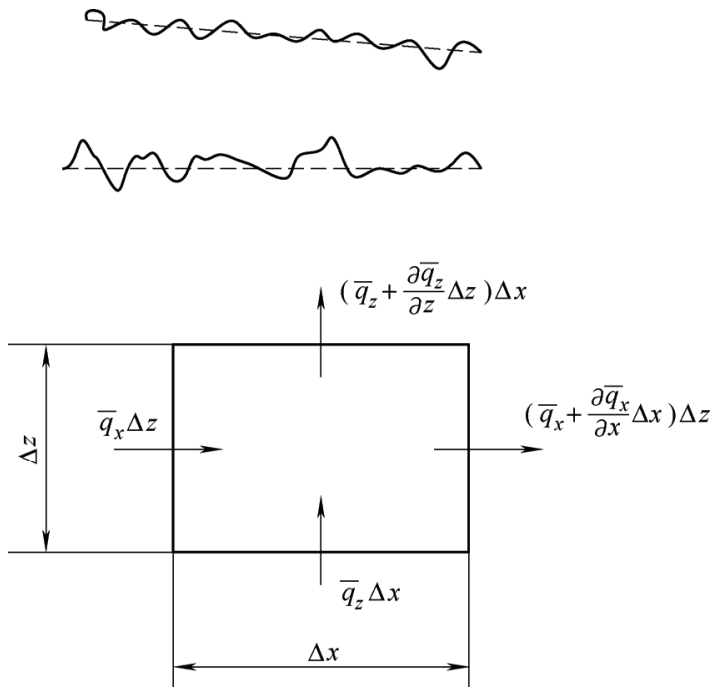

图 2 平均流动的控制体

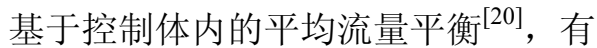

$$
\begin{gathered}
\left(\bar{q}_{x}+\frac{\partial \bar{q}_{x}}{\partial x} \Delta x\right) \Delta z-\bar{q}_{x} \Delta z+\left(\bar{q}_{z}+\frac{\partial \bar{q}_{z}}{\partial z} \Delta z\right) \Delta x-\bar{q}_{z} \Delta x= \\
-\Delta x \Delta z \frac{\partial \bar{H}}{\partial t}
\end{gathered}
$$

或

$$
\frac{\partial \bar{q}_{x}}{\partial x}+\frac{\partial \bar{q}_{z}}{\partial z}=-\frac{\partial \bar{H}}{\partial t}
$$

式中, $\bar{q}_{x}=E\left(q_{x}\right), q_{x}=\frac{V}{2} H-\frac{H^{3}}{\mu K_{x}} \frac{\partial p}{\partial x} ; \bar{q}_{z}=\mathrm{E}\left(q_{z}\right)$, $q_{z}=-\frac{H^{3}}{\mu K_{z}} \frac{\partial p}{\partial z} ; \bar{H}=\mathrm{E}(H) ; \mathrm{E}()$ 为期望算子, 由下 式定义

$$
\mathrm{E}(x)=\int_{-\infty}^{\infty} x f(x) \mathrm{d} x
$$

式中, $f(x)$ 为随机变量 $x$ 的概率密度函数。

这样，式(7)可以表示为

$$
\begin{gathered}
\frac{\partial}{\partial x}\left[\mathrm{E}\left(\frac{V}{2} H-\frac{H^{3}}{\mu K_{x}} \frac{\partial p}{\partial x}\right)\right]+ \\
\frac{\partial}{\partial z}\left[\mathrm{E}\left(-\frac{H^{3}}{\mu K_{z}} \frac{\partial p}{\partial z}\right)\right]=-\frac{\partial \mathrm{E}(H)}{\partial t}
\end{gathered}
$$

上式即为描述粗䊁表面滑动轴承整体湍流润滑的平 均 Reynolds 型湍流润滑方程。

式(8)的实际使用, 需要结合具体的粗䊁表面情 况。这里基于具体形式的粗糙表面推导可以直接使 用的一维纵向粗䊁表面、一维横向粗糙表面和各向 同性粗粘表面的平均 Reynolds 型湍流润滑方程。

以径向滑动轴承为载体的一维粗糙表面结构示 意图如图 3 所示。 


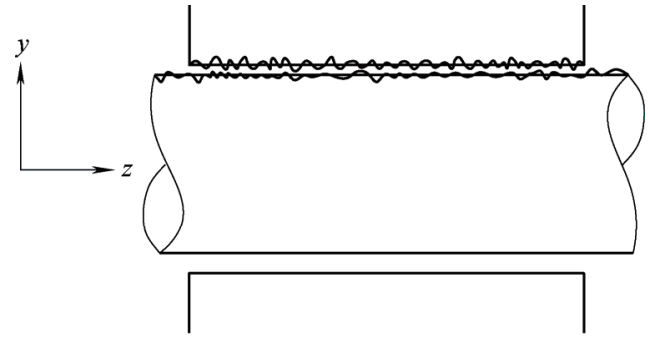

(a) 一维纵向粗糙表面

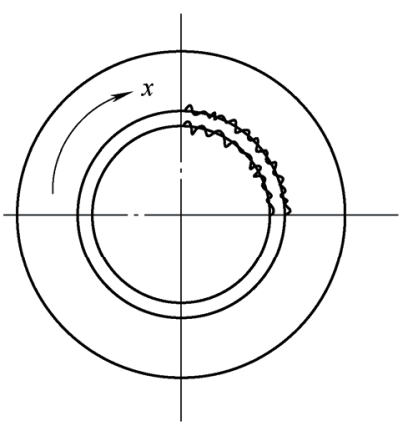

(b) 一维横向粗粘表面

图 3 径向滑动轴承表面部分粗粘结构示意图

一维纵向粗䊁表面及一维横向粗粘表面湍流润 滑模型构建的两个基本假设 ${ }^{[13]}$ 。

若 $s_{1}$ 表示平行于表面粗粘方向, $s_{2}$ 表示垂直于 表面粗粘方向。

(1) 油膜压力梯度 $\partial p / \partial s_{1}$ 是一个方差为 0 的随 机变量。

（2）垂直于表面粗糙方向的单位流量 $q_{s_{2}}$ 是一 个方差为 0 的随机变量。

\section{2 一维纵向粗糙表面的平均 Reynolds 型湍流润 滑方程}

如图 3a 所示, 一维纵向粗糙表面假设粗䊁表面 的形式为细长的微凸峰和凹谷都沿着轴颈转动方 向。这样, 油膜厚度表达式为

$$
H=h(x, z, t)+h_{s}(z, \xi)
$$

由上式可知, 油膜厚度的随机变化主要沿着轴 向方向, 则由假设(1)可知 $\partial p / \partial x$ 的方差为 0 , 而且 $\partial p / \partial x 、 H^{3}$ 和 $1 / K_{x}$ 可以看成是相互独立的随机变 量, 所以

$$
\begin{gathered}
\bar{q}_{x}=\mathrm{E}\left(\frac{V}{2} H-\frac{H^{3}}{\mu K_{x}} \frac{\partial p}{\partial x}\right)= \\
\frac{V}{2} \mathrm{E}(H)-\frac{\mathrm{E}\left(H^{3}\right) \mathrm{E}\left(1 / K_{x}\right)}{\mu} \frac{\partial \bar{p}}{\partial x}
\end{gathered}
$$

沿 $z$ 方向的单位流量为

$$
q_{z}=-\frac{H^{3}}{\mu K_{z}} \frac{\partial p}{\partial z}
$$

则 $\frac{\partial p}{\partial z}=-q_{z} \mu K_{z} / H^{3}$ 。由假设式(2)可知 $q_{z}$ 的方差为
0 , 所以

$$
\begin{gathered}
E\left(\frac{\partial p}{\partial z}\right)=\frac{\partial \bar{p}}{\partial z}=-\bar{q}_{z} \mu \mathrm{E}\left(K_{z}\right) \mathrm{E}\left(1 / H^{3}\right) \\
\bar{q}_{z}=\mathrm{E}\left(-\frac{H^{3}}{\mu K_{z}} \frac{\partial p}{\partial z}\right)=-\frac{\partial \bar{p}}{\partial z} \frac{1}{\mu \mathrm{E}\left(K_{z}\right) \mathrm{E}\left(1 / H^{3}\right)}
\end{gathered}
$$

将式(10)和(13)代入式(7), 得

$$
\begin{gathered}
\frac{\partial}{\partial x}\left[\frac{\partial \bar{p}}{\partial x} \frac{\mathrm{E}\left(H^{3}\right) \mathrm{E}\left(1 / K_{x}\right)}{\mu}\right]+ \\
\frac{\partial}{\partial z}\left[\frac{\partial \bar{p}}{\partial z} \frac{1}{\mu \mathrm{E}\left(K_{z}\right) \mathrm{E}\left(1 / H^{3}\right)}\right]=\frac{V}{2} \frac{\partial \mathrm{E}(H)}{\partial x}+\frac{\partial \mathrm{E}(H)}{\partial t}
\end{gathered}
$$

这便是一维纵向粗糙表面径向滑动轴承的平均 Reynolds 型湍流润滑方程。

\section{3 一维横向粗糙表面的平均 Reynolds 型湍流润 滑方程}

如图 3b 所示,一维横向粗糙表面假设粗䊁表面 的形式为细长的微凸峰和凹谷都垂直于轴颈转动方 向。这样, 油膜厚度表达式为

$$
H=h(x, z, t)+h_{s}(x, \xi)
$$

类似于一维纵向粗楉表面的处理, 由假设(1)可 得 $\partial p / \partial z$ 的方差为 0 , 而且 $\partial p / \partial z 、 H^{3}$ 和 $1 / K_{z}$ 可以 看成相互独立的随机变量, 所以

$$
\bar{q}_{z}=\mathrm{E}\left(-\frac{H^{3}}{\mu K_{z}} \frac{\partial p}{\partial z}\right)=-\frac{\mathrm{E}\left(H^{3}\right) \mathrm{E}\left(1 / K_{z}\right)}{\mu} \frac{\partial \bar{p}}{\partial z}
$$

沿 $x$ 方向的单位流量为

$$
q_{x}=\frac{V}{2} H-\frac{H^{3}}{\mu K_{x}} \frac{\partial p}{\partial x}
$$

则 $\frac{\partial p}{\partial x}=\left(\frac{V}{2} H-q_{x}\right) \frac{\mu K_{x}}{H^{3}}=\left(\frac{V}{2} \frac{1}{H^{2}}-\frac{q_{x}}{H^{3}}\right) \mu K_{x}$ 。由假 设(2)可知 $q_{x}$ 的方差为 0 , 所以

$$
\begin{gathered}
\mathrm{E}\left(\frac{\partial p}{\partial x}\right)=\frac{\partial \bar{p}}{\partial x}= \\
{\left[\frac{V}{2} \mathrm{E}\left(1 / H^{2}\right)-\bar{q}_{x} \mathrm{E}\left(1 / H^{3}\right)\right] \mu E\left(K_{x}\right)} \\
\bar{q}_{x}=\frac{V}{2} \frac{\mathrm{E}\left(1 / H^{2}\right)}{\mathrm{E}\left(1 / H^{3}\right)}-\frac{\partial \bar{p}}{\partial x} \frac{1}{\mu \mathrm{E}\left(1 / H^{3}\right) \mathrm{E}\left(K_{x}\right)}
\end{gathered}
$$

将式(16)和(19)代入式(7), 得

$$
\frac{\partial}{\partial x}\left[\frac{\partial \bar{p}}{\partial x} \frac{1}{\mu \mathrm{E}\left(1 / H^{3}\right) \mathrm{E}\left(K_{x}\right)}\right]+
$$

$\frac{\partial}{\partial z}\left[\frac{\partial \bar{p}}{\partial z} \frac{\mathrm{E}\left(H^{3}\right) \mathrm{E}\left(1 / K_{z}\right)}{\mu}\right]=\frac{V}{2} \frac{\partial}{\partial x} \frac{\mathrm{E}\left(1 / H^{2}\right)}{\mathrm{E}\left(1 / H^{3}\right)}+\frac{\partial \mathrm{E}(H)}{\partial t}$

这便是一维横向粗粘表面径向滑动轴承的平均 Reynolds 型湍流润滑方程。 


\section{4 各向同性粗粮表面的平均 Reynolds 型湍流润 滑方程}

由于各向同性的粗䊁表面没有方向性特征, 由 假设(1)可知: 沿圆周方向, $\partial p / \partial x$ 的方差为 0 , $\partial p / \partial x 、 H^{3}$ 及 $1 / K_{x}$ 可以看成相互独立的随机变量; 沿轴向方向, $\partial p / \partial z$ 的方差为 $0, \partial p / \partial z 、 H^{3}$ 及 $1 / K_{z}$ 可以看成是相互独立的随机变量。所以

$$
\begin{gathered}
\bar{q}_{x}=\mathrm{E}\left(\frac{V}{2} H-\frac{H^{3}}{\mu K_{x}} \frac{\partial p}{\partial x}\right)= \\
\frac{V}{2} \mathrm{E}(H)-\frac{\mathrm{E}\left(H^{3}\right) \mathrm{E}\left(1 / K_{x}\right)}{\mu} \frac{\partial \bar{p}}{\partial x} \\
\bar{q}_{z}=\mathrm{E}\left(-\frac{H^{3}}{\mu K_{z}} \frac{\partial p}{\partial z}\right)=-\frac{\mathrm{E}\left(H^{3}\right) \mathrm{E}\left(1 / K_{z}\right)}{\mu} \frac{\partial \bar{p}}{\partial z}
\end{gathered}
$$

将式(21)和(22)代入式(7), 得

$$
\begin{gathered}
\frac{\partial}{\partial x}\left[\frac{\partial \bar{p}}{\partial x} \frac{\mathrm{E}\left(H^{3}\right) \mathrm{E}\left(1 / K_{x}\right)}{\mu}\right]+ \\
\frac{\partial}{\partial z}\left[\frac{\partial \bar{p}}{\partial z} \frac{\mathrm{E}\left(H^{3}\right) \mathrm{E}\left(1 / K_{z}\right)}{\mu}\right]=\frac{V}{2} \frac{\partial \mathrm{E}(H)}{\partial x}+\frac{\partial \mathrm{E}(H)}{\partial t}
\end{gathered}
$$

这便是各向同性粗粘表面径向滑动轴承的平均 Reynolds 型湍流润滑方程。

\section{5 湍流润滑方程中油膜厚度函数和湍流润滑方 程系数函数的计算}

上述平均 Reynolds 型湍流润滑方程式(14)、(20) 和(23)的求解需要确定方程中的油膜厚度函数和湍 流润滑方程系数函数。

令随机量 $f\left(h_{s}\right)$ 为油膜厚度分量 $h_{s}$ 的概率密度函 数。本文研究 Reynolds 型粗精表面, 假定表面的粗 糙高度分布密度曲线符合 Gauss 概率密度分布曲 线, 则随机变量 $h_{s}$ 的期望为 0 , 方差为 $\sigma^{2}$, 即

$$
\begin{gathered}
\mathrm{E}\left(h_{s}\right)=\int_{-\infty}^{\infty} h_{s} f\left(h_{s}\right) \mathrm{d} h_{s}=0 \\
\mathrm{E}\left(h_{s}^{2}\right)=\int_{-\infty}^{\infty} h_{s}^{2} f\left(h_{s}\right) \mathrm{d} h_{s}=\sigma^{2}
\end{gathered}
$$

因此

$$
\begin{gathered}
\mathrm{E}(H)=\int_{-\infty}^{\infty}\left(h+h_{s}\right) f\left(h_{s}\right) \mathrm{d} h_{s}=h+\mathrm{E}\left(h_{s}\right)=h \\
\mathrm{E}\left(H^{3}\right)=\int_{-\infty}^{\infty}\left(h+h_{s}\right)^{3} f\left(h_{s}\right) \mathrm{d} h_{s}
\end{gathered}
$$

即

$$
\begin{gathered}
\mathrm{E}\left(H^{3}\right)=h^{3}+3 h^{2} \int_{-\infty}^{\infty} h_{s} f\left(h_{s}\right) \mathrm{d} h_{s}+ \\
3 h \int_{-\infty}^{\infty} h_{s}^{2} f\left(h_{s}\right) \mathrm{d} h_{s}+\int_{-\infty}^{\infty} h_{s}^{3} f\left(h_{s}\right) \mathrm{d} h_{s}
\end{gathered}
$$

由于 $h_{s}$ 是对称的, 则 $\int_{-\infty}^{\infty} h_{s}^{3} f\left(h_{s}\right) \mathrm{d} h_{s}=0$, 所以

$$
\mathrm{E}\left(H^{3}\right)=h^{3}+3 h \sigma^{2}=h^{3}\left(1+3 \frac{\sigma^{2}}{h^{2}}\right)
$$

$$
\begin{aligned}
& \text { 令 } A_{1}=a_{1} R e^{b_{1}}, A_{2}=a_{2} R e^{b_{2}} \text {, 则 } \\
& \mathrm{E}\left(K_{x}\right)=\int_{-\infty}^{\infty}\left(12+A_{1}\left(\frac{H}{h}\right)^{b_{1}}\right) f\left(h_{s}\right) \mathrm{d} h_{s}
\end{aligned}
$$

即

$$
\mathrm{E}\left(K_{x}\right)=12+A_{1} \int_{-\infty}^{\infty}\left(1+\frac{h_{s}}{h}\right)^{b_{1}} f\left(h_{s}\right) \mathrm{d} h s
$$

若润滑区域规定在流体动力润滑领域, 即两相 对运动表面无直接接触, 则 $\left|\frac{h_{s}}{h}\right|<1$ 。基于计算和精 度考量, 将 $\mathrm{E}\left(K_{x}\right)$ 等式右边积分中的被积函数部分用 Taylor 级数展开并取前三项 ${ }^{[13]}$, 那么

$$
\begin{gathered}
\int_{-\infty}^{\infty}\left(1+\frac{h_{s}}{h}\right)^{b_{1}} f\left(h_{s}\right) \mathrm{d} h_{s}= \\
\int_{-\infty}^{\infty}\left(1+b_{1} \frac{h_{s}}{h}+\frac{b_{1}\left(b_{1}-1\right)}{2}\left(\frac{h_{s}}{h}\right)^{2}\right) f\left(h_{s}\right) \mathrm{d} h_{s}
\end{gathered}
$$

则有

$$
\mathrm{E}\left(K_{x}\right)=12+A_{1}\left[1+\frac{b_{1}\left(b_{1}-1\right)}{2 h^{2}} \sigma^{2}\right]
$$

同理

$$
\mathrm{E}\left(K_{z}\right)=12+A_{2}\left[1+\frac{b_{2}\left(b_{2}-1\right)}{2 h^{2}} \sigma^{2}\right]
$$

相应地

$$
\begin{gathered}
\mathrm{E}\left(1 / H^{2}\right)=\frac{1}{h^{2}} \int_{-\infty}^{\infty} \frac{f\left(h_{s}\right)}{\left(1+\frac{h_{s}}{h}\right)^{2}} \mathrm{~d} h_{s}= \\
\frac{1}{h^{2}}\left(1+\frac{-2(-2-1)}{2} \frac{\sigma^{2}}{h^{2}}\right)
\end{gathered}
$$

即

$$
\begin{gathered}
\mathrm{E}\left(1 / H^{2}\right)=\frac{1}{h^{2}}\left(1+3 \frac{\sigma^{2}}{h^{2}}\right) \\
\mathrm{E}\left(1 / H^{3}\right)=\frac{1}{h^{3}} \int_{-\infty}^{\infty} \frac{f\left(h_{s}\right)}{\left(1+\frac{h_{s}}{h}\right)^{3}} \mathrm{~d} h_{s}= \\
\frac{1}{h^{3}}\left(1+\frac{-3(-3-1)}{2} \frac{\sigma^{2}}{h^{2}}\right)
\end{gathered}
$$

即

$$
\begin{gathered}
\mathrm{E}\left(1 / H^{3}\right)=\frac{1}{h^{3}}\left(1+6 \frac{\sigma^{2}}{h^{2}}\right) \\
\mathrm{E}\left(1 / K_{x}\right)=\int_{-\infty}^{\infty} 1 /\left(12+A_{1}\left(\frac{H}{h}\right)^{b_{1}}\right) f\left(h_{s}\right) \mathrm{d} h_{s}= \\
\frac{1}{12+A_{1}}+\left[\frac{A_{1}\left(b_{1}-b_{1}^{2}\right)}{2\left(A_{1}+12\right)^{2}}+\frac{A_{1}^{2} b_{1}^{2}}{\left(A_{1}+12\right)^{3}}\right] \frac{\sigma^{2}}{h^{2}}
\end{gathered}
$$




$$
\begin{gathered}
\mathrm{E}\left(1 / K_{z}\right)=\int_{-\infty}^{\infty} 1 /\left[12+A_{2}\left(\frac{H}{h}\right)^{b_{2}}\right] f\left(h_{s}\right) \mathrm{d} h_{s}= \\
\frac{1}{12+A_{2}}+\left[\frac{A_{2}\left(b_{2}-b_{2}{ }^{2}\right)}{2\left(A_{2}+12\right)^{2}}+\frac{A_{2}{ }^{2} b_{2}{ }^{2}}{\left(A_{2}+12\right)^{3}}\right] \frac{\sigma^{2}}{h^{2}}
\end{gathered}
$$

利用式(26) (33)可以方便地计算油膜厚度函 数和湍流润滑方程系数函数, 从而进行平均 Reynolds 型湍流润滑方程式(14)、(20)和(23)的求解。

\section{6 轴承性能参数}

图 4 为轴承结构示意图, 其中 $e$ 为偏心距, $\theta$ 为角坐标, $\psi$ 为偏位角。
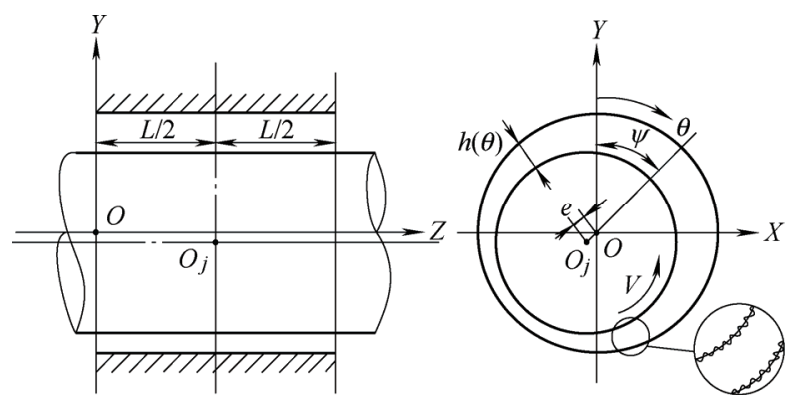

图 4 一维横向粗糙表面径向滑动轴承结构示意图

\subsection{1 轴承承载力}

如图 4 所示, 轴承承载力在 $X$ 和 $Y$ 坐标轴方向 的分量为

$$
\begin{gathered}
F_{X}=-\int_{0}^{L} \int_{0}^{2 \pi} \bar{p} R \sin \theta \mathrm{d} \theta \mathrm{d} z \\
F_{Y}=-\int_{0}^{L} \int_{0}^{2 \pi} \bar{p} R \cos \theta \mathrm{d} \theta \mathrm{d} z
\end{gathered}
$$

则轴承承载力为

$$
F=\sqrt{F_{X}^{2}+F_{Y}^{2}}
$$

式中， $R$ 为轴颈半径; $L$ 为轴承宽度。

\subsection{2 摩擦因数}

轴颈表面的摩擦力为

$$
F_{j}=\int_{0}^{L} \int_{0}^{2 \pi}\left(\frac{h}{2} \frac{\partial p}{R \partial \theta}+\frac{\mu V}{h} \bar{\tau}_{c}\right) R \mathrm{~d} \theta \mathrm{d} z
$$

$R e>10000$ 时

$$
\begin{aligned}
& \bar{\tau}_{c}=1+0.00232(R e)^{0.86}\left(1-0.0602 \frac{\sigma^{2}}{h^{2}}\right) \\
& 1000 \leqslant R e \leqslant 10000 \text { 时 } \\
& \bar{\tau}_{c}=1+0.00099(\operatorname{Re})^{0.96}\left(1-0.0192 \frac{\sigma^{2}}{h^{2}}\right)
\end{aligned}
$$

$R e<1000$ 时: $\bar{\tau}_{c}=1$ 。

轴颈表面的摩擦因数为

$$
\mu_{j}=\frac{F_{j}}{F}
$$

\section{2 模型验证}

由于目前的公开发表中仅有均匀各向同性粗糙 表面滑动轴承的湍流润滑研究, 所以使用本文提出 的各向同性粗粘表面径向滑动轴承随机湍流润滑理 论模型的分析计算结果与文献[12]中的均匀各向同 性粗粘表面滑动轴承湍流润滑试验结果进行对比, 验证模型的正确性。

表 2 为文献[12]试验研究使用的 I 型、II 型径向 滑动轴承的结构参数, 其中 $D$ 为轴颈直径, $C$ 为基 于轴颈表面粗䊁度凹谷底部和轴承孔内表面粗糙度 凹谷底部的平均径向间隙, $k_{s b}$ 为轴承孔内表面的均 匀表面粗楉高度, $k_{s j}$ 为轴颈表面的均匀表面粗糙高 度, $\sigma$ 为基于轴颈表面和轴承孔内表面的综合均方 根粗粘度。

\section{表 2 试验轴承结构参数}

\begin{tabular}{ccc}
\hline \multirow{2}{*}{ 结构参数 } & \multicolumn{2}{c}{ 轴承类型 } \\
\cline { 2 - 3 } & $\mathrm{I}$ 型 & $\mathrm{II}$ 型 \\
\hline $\mathrm{nyy} D / \mathrm{m}$ & $7.0 \times 10^{-2}$ & $7.0 \times 10^{-2}$ \\
$L / \mathrm{m}$ & $7.0 \times 10^{-2}$ & $7.0 \times 10^{-2}$ \\
$C / \mathrm{m}$ & $2.51 \times 10^{-4}$ & $3.15 \times 10^{-4}$ \\
$k_{s b} / \mathrm{m}$ & $1.0 \times 10^{-5}$ & $7.1 \times 10^{-5}$ \\
$k_{s j} / \mathrm{m}$ & $0.04 \times 10^{-6}$ & $0.19 \times 10^{-6}$ \\
$\sigma / \mathrm{m}$ & $5.0 \times 10^{-6}$ & $3.55 \times 10^{-5}$ \\
\hline
\end{tabular}

文献[12]的轴承试验结果使用轴承性能参数 索默菲德数 $S$ 表示, 轴承润滑剂流动状态使用 $R e^{*}$ 表征。

$$
S=\frac{\mu L V R^{2}}{\pi W C^{2}} \quad R e^{*}=\frac{\rho C V}{\mu}
$$

式中, $\mu$ 为水的动力黏度; $W$ 为载荷; $V$ 为轴颈表面 速度; $\rho$ 为水的密度。

以文献[12]中的 I 型、II 型径向滑动轴承为研究 对象, 结合式(26)、(27)、(32)和(33), 应用有限差 分法求解本文提出的各向同性粗糙表面径向滑动轴 承的平均 Reynolds 型湍流润滑方程(23)进行理论分 析计算。

理论分析计算结果与相应试验结果的比较如图 5 所示。其中, C 模型计算值和 NPE 模型计算值分 别为采用表 1 中 Constantinescu 湍流润滑模型和 Ng-Pan-Elrod 湍流润滑模型确定式(32)和(33)参数 $a_{1} 、 a_{2} 、 b_{1}$ 和 $b_{2}$ 取值情况下的理论分析计算值。

由图 5 的对比可见，理论分析计算值与试验值 的一致性良好，验证了本文提出的粗䊁表面湍流润 滑随机理论模型的正确性。 


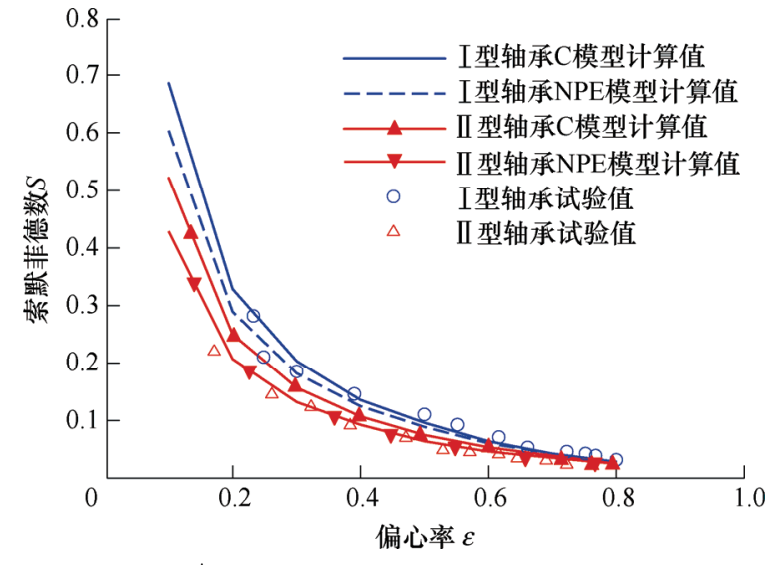

图 $5 R e^{*}=3500$ 时索默菲德数随偏心率的变化

\section{3 分析与讨论}

\section{1 与其他各向同性粗糙表面湍流润滑模型的比较}

以表 2 中 I 型径向滑动轴承为研究对象, 图 6 所示为本文提出的粗糙表面随机湍流润滑模型与文 献[12]中 Hashimoto 提出的各向同性粗精表面湍流 润滑模型( $\mathrm{H}$ 模型)的计算结果和相应试验结果。

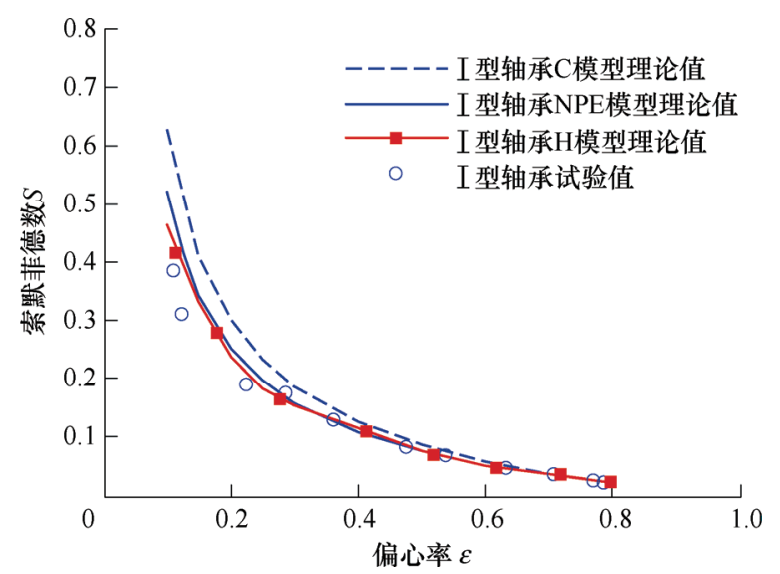

图 $6 R e^{*}=4500$ 时索默菲德数随偏心率的变化

经对比发现, 本文提出的粗粘表面湍流润滑随 机模型及 Hashimoto 各向同性粗糙表面湍流润滑模 型的理论计算值与试验值的一致性较好, 再次验证 了本文提出的粗糙表面湍流润滑随机模型的正确 性。而且, NPE 模型计算结果与 $\mathrm{H}$ 模型计算结果吻 合性较好, $\mathrm{C}$ 模型计算结果相比 $\mathrm{H}$ 模型计算结果仅 在较小偏心率 $(\varepsilon \leqslant 0.4)$ 下有一定的偏差。

\section{2 各向异性粗糙表面径向滑动轴承的湍流润滑} 分析

以表 2 中 I 型径向滑动轴承为研究对象, 应用 本文提出的一维横向粗糙轴承的随机湍流润滑模型 (20)和一维纵向粗糙轴承的随机湍流润滑模型(14) 进行粗粘表面方向性特征的对比分析。轴承承载力 和摩擦因数的结果如图 7 和图 8 所示。

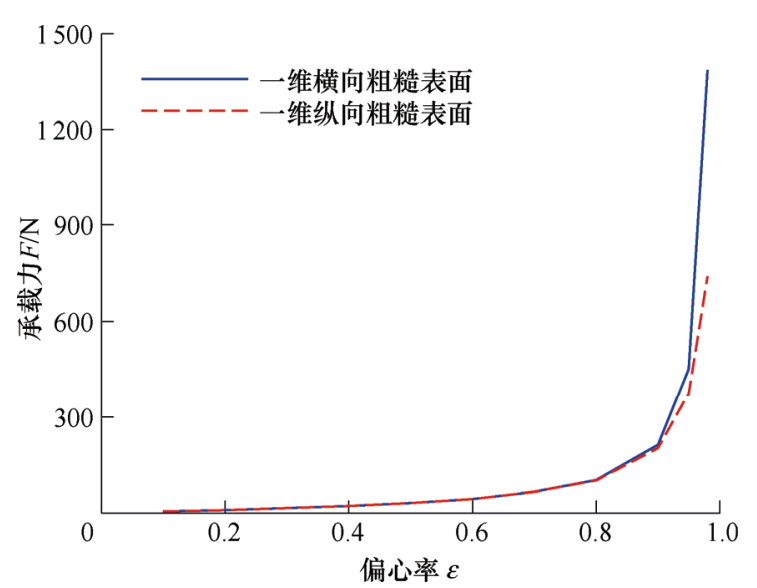

图 $7 R e^{*}=3500$ 时承载力随偏心率的变化

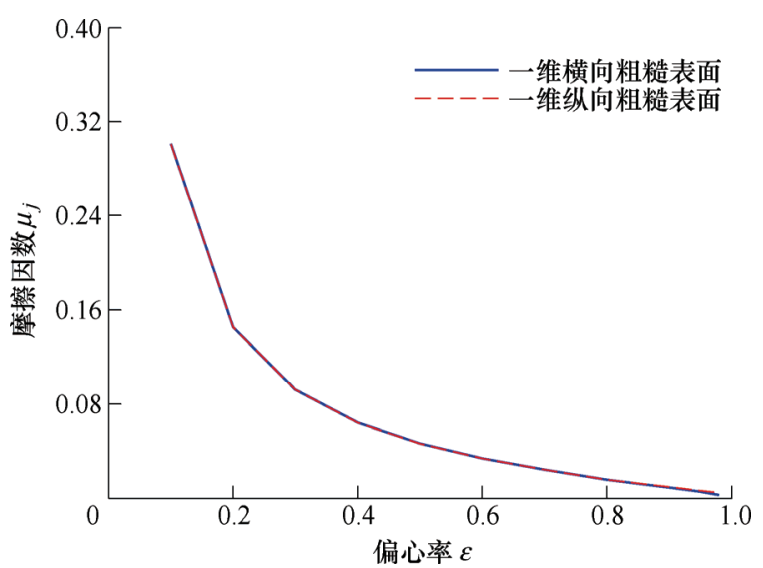

图 $8 R e^{*}=3500$ 时摩擦因数随偏心率的变化

由图 7 可见一维横向粗糙表面轴承和一维纵向 粗粘表面轴承的承载力仅在较大的偏心率 $(\varepsilon>0.9)$ 下 出现明显差异，在其余偏心率下基本相同。

由图 8 可知一维横向粗糙表面轴承和一维纵向 粗粘表面轴承的摩擦因数在各偏心率下基本相同。

\section{3 模型的便利性和适用性}

以一维纵向粗粘表面径向滑动轴承的平均 Reynolds 型湍流润滑方程式(14)为讨论对象。

当 $h / \sigma$ 比较大时, 表面粗䊁的影响可以忽略, 方程式(14)可简化为

$$
\frac{\partial}{\partial x}\left[\frac{h^{3}}{\mu k_{x}} \frac{\partial p}{\partial x}\right]+\frac{\partial}{\partial z}\left[\frac{h^{3}}{\mu k_{z}} \frac{\partial p}{\partial z}\right]=\frac{V}{2} \frac{\partial h}{\partial x}+\frac{\partial h}{\partial t}
$$

其与光滑表面的径向滑动轴承湍流润滑方程式(1) 相同。

当 $R e$ 数较小时,润滑剂的流动状态由湍流转变 为层流，方程式(14)可简化为

$$
\begin{gathered}
\frac{\partial}{\partial x}\left[\frac{\partial \bar{p}}{\partial x} \frac{E\left(H^{3}\right)}{12 \mu}\right]+\frac{\partial}{\partial z}\left[\frac{\partial \bar{p}}{\partial z} \frac{1}{12 \mu E\left(1 / H^{3}\right)}\right]= \\
\frac{V}{2} \frac{\partial h}{\partial x}+\frac{\partial h}{\partial t}
\end{gathered}
$$

其与 Christensen 的一维纵向粗糙表面径向滑动 轴承的层流润滑方程 ${ }^{[13]}$ 相同。 
由此可见, 本文推导提出的粗糙表面湍流润滑 的随机模型可以弥补光滑表面湍流润滑模型或粗 粘表面层流润滑模型在运用中只能分别单独考虑 湍流流动效应或表面粗糙效应的不足, 为全面深入 开展湍流流动效应和表面粗粘效应的研究提供理 论支持。

再有, 与光滑表面湍流润滑理论模型式(1)相比 较, 本文推导提出的粗粘表面随机湍流润滑理论模 型式(14)、(20)和(23)的基本形式相同, 最主要不同 点仅在于增加了表征表面粗糙情况的综合均方根 粗粘度参数 $\sigma$, 因而随机湍流润滑理论模型方程的 数值求解过程与光滑表面湍流润滑理论模型方程 的数值求解过程也基本相同, 所以本文提出的粗粘 表面湍流润滑随机模型应用于粗糙表面径向滑动轴 承的湍流润滑分析时可以基本不增加问题求解的复 杂性。

此外, 滑块和止推轴承等摩擦副零件与径向滑 动轴承基于同样的润滑原理, 因此本文推导提出的 一维纵向粗糙表面湍流润滑模型方程、一维横向粗 糙表面湍流润滑模型方程和各向同性粗粘表面湍流 润滑模型方程也同样适用于滑块和止推轴承等摩擦 副零件的湍流润滑分析。

\section{4 结论}

(1) 提出了适用于粗䊁表面径向滑动轴承湍流 润滑分析的随机湍流润滑理论模型, 并验证了模型 的正确性。

(2) 随机湍流润滑理论模型可以适用于具有方 向性特征的粗粮表面湍流润滑分析, 弥补了当前考 虑表面粗糙影响的湍流润滑模型只能适用于各向同 性粗糙表面湍流润滑分析的不足。

(3) 随机湍流润滑理论模型可以非常方便地应 用于粗粮表面径向滑动轴承的湍流润滑分析。

(4) 随机湍流润滑理论模型不仅可以分析粗粘 表面径向滑动轴承的湍流润滑, 而且也可以分析粗 糙表面滑块和止推轴承等摩擦副零件的湍流润滑。

\section{参 考 文 献}

[1] 张直明, 张言羊, 谢友柏, 等. 滑动轴承的流体动力润 滑理论[M]. 北京: 高等教育出版社, 1986.

ZHANG Zhiming, ZHANG Yanyang, XIE Youbai, et al. Hydrodynamic lubrication theory of plain bearings[M]. Beijing: Higher Education Press, 1986.

[2] LÜ Fangrui, JIAO Chunxiao, TA Na, et al. Mixed-lubrication analysis of misaligned bearing
consideringturbulence[J]. Tribology International, 2018, 119: $19-26$.

[3] 宋智翔, 刘荣, 郭飞, 等. 屏蔽式核主泵水润滑可倾瓦 推力轴承推力盘的离心效应 $[\mathrm{J}]$. 机械工程学报, 2018, 54(1): 127-135.

SONG Zhixiang, LIU Ying, GUO Fei, et al. Influence of centrifugal deformation of thrust collar inwater-lubricated tilting-pad thrust bearings of nuclear canned pump[J]. Journal of Mechanical Engineering, 2018, 54(1): 127-135.

[4] TAYLOR C M, DOWSON D. Turbulent lubrication theory-application to design[J]. Journal of Lubrication Technology, 1974, 96(1): 36-46.

[5] CONSTANTINESCU V N. On turbulent lubrication[J]. Proceedings of the Institution of Mechanical Engineers, 1959, 173(1): 881-900.

[6] NG C W, PAN C H T. A linearized turbulent lubrication theory[J]. Journal of Basic Engineering, 1965, 87(3): 675-682.

[7] ELORD H G, NG C W. A theory for turbulent fluid films and its application to bearings[J]. Journal of Lubrication Technology, 1967, 89(3): 346-362.

[8] HIRS G G. A bulk-flow theory for turbulence in lubricant films[J]. Journal of Lubrication Technology, 1973, 95(2): 137-145.

[9] HASHIMOTO H, WADA S. Theoretical approach to turbulent lubrication problems including surface roughness effects[J]. Journal of Tribology, 1989, 111(1): 17-22.

[10] HASHIMOTO H , MONGKOLWONGROJN M. Adiabatic approximate solution for static and dynamic characteristics of turbulent partial journal bearings with surface roughness[J]. Journal of Tribology, 1994, 116(4): 672-680.

[11] HASHIMOTO H. Thermohydrodynamic analysis of high-speed journal bearings with surface roughness[J]. Journal of Tribology, 1996, 118(3): 698-701.

[12] HASHIMOTO H. Surface roughness effects in high-speed hydrodynamic journal bearings[J]. Journal of Tribology, 1997, 119(4): 776-780.

[13] CHRISTENSEN H. Stochastic models for hydrodynamiclubrication of rough surfaces[J]. Proceedings of the Institution of Mechanical Engineers, 1969, 184(1): 1013-1026.

[14] 装祖干. 非牛顿流体有限长粗䊅轴承分析[J]. 应用力 学学报, 1992, 9(1): 16-21.

QIU Zugan. Analysis of finite width rough journal bearing with non-Newtonian fluids[J]. Chinese Journal of Applied Mechanics, 1992, 9(2): 16-21.

[15] 震祖干, 张长松. 动载径向粗糙轴承分析 $[\mathrm{J}]$. 内燃机学 
报，1993，11(2): 159-164.

QIU Zugan , ZHANG Changsong. Analysis of dynamically loaded rough journal bearing with finite width[J]. Transactions of CSICE, 1993, 11(2): 159-164.

[16] KANE M, BOU-SAID B. Comparison of homogenization and direct techniques for the treatment of roughness in incompressible lubrication[J]. Journal of Tribology, 2004, 126(4): 733-737.

[17] LAHMAR M, BOU-SAID B, TICHY J. The homogenization method of roughness analysis in turbulent lubrication[J]. Journal of Engineering Tribology, 2013, 227(10): 1090-1100.

[18] WANG Xiaojing, ZHANG Zhiming, SUN Meili. A comparison of flow fields predicted by various turbulent lubrication models with existing measurements[J]. Journal of Tribology, 2000, 122(2): 475-477.

[19] 张直明. 论紊流润滑理论中的 Hirs 法的理论基础 [J]. 西安交通大学学报，1978，(4): 25-28.

ZHANG Zhiming. On the theoretical basis of the Hirs method in the theory of lubrication[J]. Journal of Xi'an Jiaotong University, 1978, (4): 25-28.

[20] PATIR N, CHENG H S. An average flow model for determining effects of three-dimensional roughness on partial hydrodynamic lubrication[J]. Journal of Lubrication Technology, 1978, 100(1): 12-17.

作者简介: 朱少禹, 男, 1994 年出生, 博士研究生。主要研究方向为现 代车辆设计理论与方法。

E-mail: 2434492223@qq.com

孙军(通信作者), 男, 1960 年出生, 博士, 教授, 博士研究生导师。主 要研究方向为摩擦学和现代设计理论与方法。

E-mail: sunjun_hfut@163.com 